\title{
MACULAR VITREORETINAL INTERFACE ABNORMALITIES IN HIGHLY MYOPIC EYES WITH POSTERIOR STAPHYLOMA 5-Year Follow-up
}

\author{
GUIDO RIPANDELLI, MD,* TOMMASO ROSSI, MD, $\dagger$ FABIO SCARINCI, MD, ${ }^{*}$ CECILIA SCASSA, MD,* \\ VINCENZO PARISI, MD,* MARIO STIRPE, MD*
}

Purpose: To review prevalence, long-term progression, and prognosis of vitreoretinal interface modifications in pathologic myopia with posterior staphyloma and investigate foveal sensitivity and fixation stability.

Methods: Retrospective single-institution series of 214 eyes (116 patients) with pathologic myopia, axial length $>30 \mathrm{~mm}$, and posterior staphyloma. Exclusion criteria included follow-up less than five years, incomplete records, and/or less than three optical coherence tomography or microperimetry. Patients were divided into 5 groups according to optical coherence tomography: 1) epiretinal membrane without schisis (ERM); 2) macular retinal schisis (Schisis); 3) partial thickness macular hole (PTMH); 4) full-thickness macular hole $(\mathrm{FTMH})$; and 5) posterior retinal detachment (PRD) with or without macular hole. Disease progression was defined as a visual acuity decrease of two or more lines associated to objective worsening of the optical coherence tomography and/or microperimetry.

Results: Vitreoretinal abnormalities at baseline were present in 116 of 204 patients (56.8\%) and 214 of 408 eyes (52.4\%); 98 of 116 patients (84.4\%) showed bilateral involvement. Baseline visual acuity and foveal sensitivity varied significantly with ERM performing better and PRD worse than others; PTMH and FTMH did not differ. During the 66 months of average follow-up, 33 of 214 eyes (15.4\%) required surgery and 13 of 33 eyes $(39.3 \%)$ needed reintervention. Surgery rate significantly differed among groups: $2 \%$ for ERM, $20 \%$ to $25 \%$ for Schisis, PTMH, and FTMH, and up to $50 \%$ for PRD. Progression rate of Schisis and FTMH was the same, regardless of symptoms, while macula-off PRD always required surgery. Decrease of fixation stability and foveal sensitivity correlated to need for surgery, while baseline foveal sensitivity and fixation did not.

Conclusion: Vitreoretinal interface pathology in pathologic myopia with posterior staphyloma encompasses a spectrum of conditions whose baseline functionality, prognosis, rate, and amount of progression vary significantly. Customized treatment for each different condition should be considered.

RETINA 32:1531-1538, 2012

$\mathrm{P}$ athologic myopia (PM) affects the sclera, choroid, Bruch membrane, retinal pigment epithelium, neurosensory retina, and the vitreous. ${ }^{1}$ Bulb elongation ${ }^{2}$ and posterior staphyloma (PS) represent the hallmark of

From the *G.B. Bietti Eye Foundation-IRCCS, Rome, Italy; and $\dagger$ Department of Ophthalmology, Oftalmico Hospital, Rome, Italy.

The authors report no proprietary or financial interest to disclose.

Reprints: Guido Ripandelli, MD, G.B. Bietti Eye FoundationIRCCS, Rome, Italy, Via Livenza, 300199 Rome, Italy; e-mail: g.ripandelli@email.it disease and are strictly correlated, with more than $70 \%$ of eyes longer than $36 \mathrm{~mm}$ presenting a prominent PS. ${ }^{3}$

All tissues involved in the staphyloma show specific alterations including vitreoschisis, epiretinal membranes, macular retinal schisis, ${ }^{4}$ macular holes $(\mathrm{MHs})^{5}$ and localized retinal detachment. ${ }^{6}$ Dismal visual prognosis has been reported in PM with PS. ${ }^{7}$

Purpose of the present article is to document the longterm progression and functional prognosis of patients with PM associated with PS and to assess whether 
microperimetry (MP) can provide valuable information in the management of such patients.

\section{Materials and Methods}

The records of highly myopic patients seen at a single institution between December 2001 and December 2004 were retrospectively reviewed. Inclusion criteria were axial length $>30 \mathrm{~mm}$, presence of PS, and the availability of optical coherence tomography (OCT) and MP at least at baseline, latest examination, and immediately before and after surgery. Patients with significant media opacity, previous ocular surgery, need for ocular surgery other than for retinal reason (glaucoma, cataract extraction, etc), age $<18$ years, and incomplete charts were excluded. Two hundred and four patients (408 eyes) met inclusion criteria, 116 of whom (214 eyes) showed vitreoretinal abnormalities within the staphyloma and were therefore included in the study.

Comprehensive visit included Early Treatment Diabetic Retinopathy Study (ETDRS) Snellen equivalent best-corrected visual acuity, anterior and posterior segment biomicroscopy, intraocular pressure, dilated indirect ophthalmoscopy, and biomicroscopy. Instrumental diagnostic procedures, such as A- and Bscan ultrasonography (Humphrey Instruments, San Leandro, CA), OCT (OCT3 Stratus; Carl Zeiss Ophthalmic Systems, Inc, Humphrey Division, Dublin, CA, before September 2007, and Cirrus Spectral Domain High Definition-Carl Zeiss Meditec, Inc, Dublin, CA, thereafter), ${ }^{5,6}$ and MP (MP1; Nidek Technologies, Padua, Italy) were performed consistently throughout the entire sample as deemed necessary.

Axial length was measured by means of A-Scan ultrasonography (HiScan; Optikon 2000, Rome, Italy). Microperimetry used a 32-stimuli grid over the central 5 degrees, 1 second between stimuli, Goldmann I stimulus size, and a bright fixation target of 1 degree. A 4-2-1 double staircase strategy was carried out, starting at the $15 \mathrm{db}$ level. Fixation was defined stable if fell $>75 \%$ of times within the inner 2 degrees, relatively unstable if $<75 \%$ were inside the 2 degree but $>75 \%$ inside 4 degrees, and unstable if $<75 \%$ fell within 4 degrees as elsewhere reported. ${ }^{8}$ Foveal sensitivity and fixation stability were calculated for the overall sample and for each group during the course of follow-up and correlated to OCT and ophthalmoscopic findings. The variation of foveal sensitivity and fixation stability between baseline examination and preoperative visit for eyes requiring surgery or end of follow-up visit for stable eyes was also recorded.

Eyes were divided into 5 groups based on ophthalmoscopic and OCT findings ${ }^{9}$ at baseline: 1) epiretinal membrane without retinal changes (ERM); 2) macular retinal schisis (Schisis) with or without ERM; 3) partial thickness macular hole (PTMH); 4) full-thickness macular hole without retinal detachment (FTMH); and 5) posterior retinal detachment (PRD) with or without MH.

Disease progression was defined as a decrease in visual acuity of 2 or more lines in 2 consecutive visits associated to objective worsening of the OCT and/or MP. Patients referring loss of vision, onset or worsening of metamorphopsia, central scotoma, and/or sudden decrease in visual acuity have been considered "symptomatic."

To evaluate disease behavior and progression patterns within groups, Schisis and FTMH have been further classified into symptomatic and asymptomatic while PRD has been divided into macula on, macula off, and associated to MHs. Vitreous status was assessed by means of indirect ophthalmoscopy, slitlamp biomicroscopy with +90-diopter lens and, when deemed necessary, B-scan ultrasound; a diagnosis of posterior vitreous detachment was made either if biomicroscopy showed Weiss ring anterior to the optic disk or if ultrasonography clearly outlined a membrane freely moving anterior to the optic nerve shadow.

For the purpose of statistical analysis, Snellen fraction visual acuity data have been converted into logarithm of the minimum angle of resolution unit. Statistical analysis used analysis of variance for metric variables and chisquare for contingency tables, whereas the nonparametric Spearman correlation test was used for fixation stability and need for surgery. Significance has been set at the 0.05 level. The study followed the tenets of the Declaration of Helsinki and received approval from the Institutional Review Board affiliated with G.B. Bietti Eye Foundation-IRCCS, Rome, Italy.

\section{Results}

\section{Baseline}

Vitreoretinal abnormalities at baseline were present in 116 of 204 originally screened patients (56.8\%) and 214 of 408 eyes (52.4\%); 98 of 116 patients $(84.4 \%)$ showed bilateral involvement. ${ }^{10}$ Study population therefore included 214 eyes of 116 patients whose demographics and baseline characteristics are reported in Table 1 . Patient's age, axial length, and grade of ametropy did not differ significantly among groups (Table 1).

Patients in ERM group had better vision and those in PRD group had worse vision than any other group at baseline while PTMH and FTMH groups did not differ significantly (Table 1). Foveal sensitivity behaved likewise with higher values in ERM patients 
Table 1. Baseline Data

\begin{tabular}{lccccccc}
\hline & ERM & Schisis & PTMH & FTMH & PRD & Significance & Total \\
\hline No. of eyes (\%) & $98(45.7)$ & $54(25.2)$ & $24(11.2)$ & $18(8.4)$ & $20(9.3)$ & & $214(100)$ \\
Mean age \pm SD (years) & $42.2 \pm 8.1$ & $41.1 \pm 7.6$ & $40.3 \pm 7.5$ & $42.2 \pm 8.4$ & $39.9 \pm 9.1$ & n.s. & $41.4 \pm 9.7$ \\
Sex (\%, males/females) & $48 / 52$ & $48 / 52$ & $47 / 53$ & $41 / 59$ & $42 / 58$ & n.s. & $46 / 54$ \\
Follow-up (months) & $65.6 \pm 5.6$ & $64.1 \pm 6.2$ & $68.6 \pm 7.1$ & $64.6 \pm 4.2$ & $65.1 \pm 4.4$ & n.s. & $66.6 \pm 6.7$ \\
Axial length (mm) & $30-33.6$ & $30.7-34.4$ & $31.2-33.9$ & $30.8-33.7$ & $31.9-34.2$ & n.s. & $30-34.4$ \\
Myopia (-dpt) & $18-28$ & $18-29$ & $18-30$ & $19-29$ & $19-30$ & n.s. & $18-30$ \\
Foveal sensitivity (dB) & $15.5 \pm 2.5$ & $13.8 \pm 3.0$ & $14.7 \pm 2.9$ & $12.7 \pm 2.2$ & $9.2 \pm 4.8$ & $P<0.001^{*}$ & $14.4 \pm 3.1$ \\
$\quad$ at baseline & & & & & & & \\
LogMAR at baseline ( \pm SD) & $0.25 \pm 0.11$ & $0.35 \pm 0.11$ & $0.36 \pm 0.12$ & $0.43 \pm 0.14$ & $1.29 \pm 0.57$ & $P<0.001$ & $0.3 \pm 0.2$ \\
\hline
\end{tabular}

ERM better than Schisis and PRD; PRD worse than any other group; and PTMH did not show significant difference to any group. * Statistical significance of foveal sensitivity at baseline among groups (at the 0.01 level).

n.s., not significant.

compared with other groups, except PTMH. Eyes rated the worst in PRD group $(P<0.001$ against all other groups); Schisis and FTMH groups performed better than ERM and PRD while PTMH only differed from PRD. Fixation stability at baseline rated significantly worse in PRD and FTMH, as opposed to ERM and Schisis eyes $(P<0.001)$. Posterior vitreous detachment was present in 48 of 214 eyes (22.4\%), while the remaining 166 of 214 eyes (77.6\%) showed a vitreous lacuna within the staphyloma.

\section{Follow-up}

During the 66 months of average follow-up, 33 of 214 eyes (15.4\%) underwent surgery and 13 of 33 eyes $(39.3 \%)$ required a second operation, with surgery rate varying significantly between $2 \%$ for ERM to $20 \%$ to $25 \%$ for Schisis, PTMH, and FTMH, and up to $50 \%$ for the PRD (Table 2). Details of patients who did not require surgery are reported in Table 3 ; the significant differences at baseline compared with surgical patients were PTMH and PRD logarithm of the minimum angle of resolution and foveal sensitivity.

Chances of progression for symptomatic and asymptomatic patients were the same for Schisis and FTMH groups, whereas being macula off or having a $\mathrm{MH}$ rose the chances of undergoing surgery in the PRD group. The need for surgery significantly correlated to a decrease of both fixation stability and foveal sensitivity while baseline foveal sensitivity and fixation stability did not matter (Table 4). Selected cases of patients whose disease progressed to require surgery are reported hereafter, to exemplify the diverse evolution of vitreoretinal abnormalities associated to pathologic myopia.

\section{ERM Patient No. 16}

A 42-year-old man with -22 diopters refraction, 16 dB foveal sensitivity, and baseline acuity of $20 / 25$ returned 18 months after the initial examination complaining of metamorphopsia and loss of vision. Visual acuity dropped to 20/200 and MP to $9 \mathrm{~dB}$ because of the development of foveal Schisis. The patient underwent pars plana vitrectomy (PPV), ERM and internal limiting membrane (ILM) peeling, SF6 tamponade with good anatomical and functional restoration; 6 months after surgery, vision was 20/30 and foveal sensitivity was $14 \mathrm{~dB}$.

\section{Schisis Patient No 43}

A 37-year-old woman with -26 diopters of myopia, $20 / 40$ vision, and $14 \mathrm{~dB}$ at baseline started complaining of "blurred" vision 8 months after the initial examination. Vision was unchanged while foveal sensitivity dropped to $12 \mathrm{~dB}$ with stable fixation (Figure 1A). Optical coherence tomography showed foveal thickness increase (Figure 1B). Six months later, vision also had decreased to $20 / 100$ and sensitivity measured $10 \mathrm{~dB}$ in fovea with relatively stable fixation (Figure 1C); OCT showed a further slight increase in foveal thickness (Figure 1D). The patient underwent PPV, ILM peeling, and SF6 tamponade, achieving reduction of foveal thickness but minimal visual benefit, unchanged foveal sensitivity, and fixation instability. A second surgical procedure with episcleral macular buckle (EMB) performed 2 months later reached foveal adhesion and allowed fixation stability.

\section{PTMH Patient No. 11}

A 45-year-old woman with 20/40 vision at baseline (refraction -19 diopters; foveal sensitivity at baseline $13 \mathrm{~dB}$ ) experienced "blurred vision" 1 month after the initial examination, and despite unchanged visual acuity, MP revealed decreased foveal sensitivity $(10 \mathrm{~dB})$. Three months later, visual acuity was 20/60, foveal sensitivity was $9 \mathrm{~dB}$, fixation was relatively stable, and OCT showed marked foveal thickening and 
Table 2. Details of Patients Requiring Surgery in the Course of Follow-up

\begin{tabular}{|c|c|c|c|c|c|c|c|c|c|}
\hline Group & \multicolumn{2}{|c|}{ Surgery } & $\begin{array}{l}\text { Baseline } \\
\text { LogMAR }\end{array}$ & $\begin{array}{l}\text { Baseline } \\
\text { MP1 (dB) }\end{array}$ & \multicolumn{2}{|c|}{$\begin{array}{l}\text { Elapsed Time Range } \\
\text { (Months) }\end{array}$} & $\begin{array}{l}\text { Preoperative } \\
\text { LogMAR }\end{array}$ & $\begin{array}{l}\text { Preoperative } \\
\text { MP1 (dB) }\end{array}$ & $\begin{array}{c}\text { Preoperative } \\
\text { Fixation }\end{array}$ \\
\hline ERM & \multicolumn{2}{|c|}{$2 / 98(2 \%)$} & 0.2 & 15.4 & $18-24$ & & \multirow{2}{*}{$\begin{array}{c}0.8 \\
-\end{array}$} & 9.8 & $2 \mathrm{R}$ \\
\hline Schisis & \multicolumn{2}{|c|}{$13 / 54(24 \%)$} & 0.3 & 13.8 & $1-36$ & & & - & - \\
\hline Asymptomatic & \multicolumn{2}{|c|}{$6 / 40^{*}(15 \%)$} & 0.2 & 15.3 & $2-9$ & & 0.6 & \multirow{2}{*}{$\begin{array}{r}10.8 \\
5.2\end{array}$} & $4 \mathrm{~S}, 2 \mathrm{R}$ \\
\hline Symptomatic & \multirow{2}{*}{\multicolumn{2}{|c|}{$7 / 14^{*}(50 \%)$}} & 0.3 & 10.3 & $14-36$ & & 1.10 & & $2 S, 5 R$ \\
\hline PTMH & & & 0.4 & 14.7 & $1-5$ & & 0.8 & 8.9 & $5 \mathrm{R}$ \\
\hline FTMH & \multicolumn{2}{|c|}{$3 / 18$ (16.6\%) } & 0.4 & 12.7 & $11-42$ & & - & - & - \\
\hline Asymptomatic & \multicolumn{2}{|c|}{$\quad 2 / 9(22.2 \%)$} & 0.2 & 14.0 & $11-23$ & & \multirow{2}{*}{$\begin{array}{l}0.7 \\
0.7\end{array}$} & 9.8 & $2 \mathrm{R}$ \\
\hline Symptomatic & \multirow{2}{*}{\multicolumn{2}{|c|}{$1 / 9(11.1 \%)$}} & 0.4 & 11.5 & 42 & & & \multirow{2}{*}{7.8} & $\mathrm{R}$ \\
\hline PRD & & & - & - & - & & $\begin{array}{l}0.1 \\
-\end{array}$ & & - \\
\hline Fovea on & \multirow{2}{*}{\multicolumn{2}{|c|}{$\begin{array}{l}1 / 11(9 \%) \\
4 / 4(100 \%)\end{array}$}} & 0.2 & 14.3 & 3 & & 1 & 5.0 & $\mathrm{R}$ \\
\hline Fovea off & & & 0.9 & 4.9 & 0 & & 0.9 & 4.9 & $4 \mathrm{R}$ \\
\hline $\mathrm{MH}+\mathrm{PRD}$ & \multicolumn{2}{|c|}{$5 / 5(100 \%)$} & 1.3 & 4.5 & 0 & & 1.3 & 4.4 & $5 U$ \\
\hline Group & Surgery & $\begin{array}{r}\text { Postor } \\
\text { log }\end{array}$ & $\begin{array}{l}\text { erative } P \\
\text { MAR }\end{array}$ & $\begin{array}{l}\text { Postoperative } \\
\text { MP1 (dB) }\end{array}$ & $\begin{array}{l}\text { Postoperative } \\
\text { Fixation }\end{array}$ & $\begin{array}{l}\text { Secono } \\
\text { Surgery }\end{array}$ & $\begin{array}{l}\text { nd } \\
\text { ry }\end{array}$ & $\begin{array}{l}\text { r Second } \\
y(d B)\end{array}$ & $\begin{array}{l}\text { Fixation After } \\
\text { econd Surgery }\end{array}$ \\
\hline ERM & $2 \mathrm{PPV}$ & & 2 & 14.3 & $1 \mathrm{R}, 1 \mathrm{~S}$ & - & & - & - \\
\hline Schisis & - & & - & - & - & - & & - & - \\
\hline Asymptomatic & $6 \mathrm{PPV}$ & & .2 & 13.3 & $3 \mathrm{~S}, 3 \mathrm{R}$ & $3 \mathrm{EMB}$ & & .3 & $2 \mathrm{~S}, 1 \mathrm{R}$ \\
\hline Symptomatic & 7 PPV & & .6 & 8.6 & $5 S, 2 R$ & $3 \mathrm{EMB}$ & & 4 & $2 S, 1 R$ \\
\hline PTMH & 5 PPV & & .4 & 11.3 & $2 S, 3 R$ & $2 \mathrm{EMB}$ & & 2 & $1 \mathrm{~S}, 1 \mathrm{R}$ \\
\hline FTMH & - & & - & - & - & - & & & - \\
\hline Asymptomatic & $2 \mathrm{PPV}$ & & .7 & 10.0 & $1 \mathrm{~S}, 1 \mathrm{R}$ & - & & - & - \\
\hline Symptomatic & PPV & & .4 & 11.2 & $S$ & - & & - & - \\
\hline PRD & - & & - & - & - & - & & - & - \\
\hline Fovea on & PPV & & .4 & 10.5 & S & - & & - & - \\
\hline Fovea off & 4 PPV & & .5 & 9.7 & $1 S, 3 R$ & $2 \mathrm{EMB}$ & & .8 & $1 \mathrm{~S}, 1 \mathrm{R}$ \\
\hline $\mathrm{MH}+\mathrm{PRD}$ & 5 PPV & & 8 & 9.6 & $2 R, 3 U$ & $3 \mathrm{EMB}$ & & .1 & $1 \mathrm{~S}, 2 \mathrm{R}$ \\
\hline
\end{tabular}

${ }^{*}$ Difference between rate of progression in symptomatic and asymptomatic Schisis patients is significant $(P=0.008)$, while it is not for FTMH group.

LogMAR, logarithm of the minimum angle of resolution.

schisis-like changes. Pars plana vitrectomy with ILM peel and SF6 tamponade restored good anatomy and stable fixation.

\section{FTMH Patient No. 4}

A 50-year-old man (sph -22; visual acuity at baseline $20 / 50$, foveal sensitivity $13 \mathrm{~dB}$, with stable fixation and no evidence of dense central scotoma despite the OCT finding) presented for a routine examination, being totally asymptomatic. Baseline OCT revealed the presence of FTMH and adherent ERM. Twentyfour months later, visual acuity had progressively dropped to 20/80 and MP1 measured $8 \mathrm{~dB}$ with relatively stable fixation, central macular scotoma, and worsened OCT. The patient underwent PPV, accurate cortical vitreous peel, ILM peel, and SF6 tamponade. Six months later, visual acuity and foveal sensitivity did not change substantially (20/80 visual acuity and $9 \mathrm{~dB}$, respectively) despite a relatively favorable anatomical outcome and closed MH. Foveal fixation became relatively stable after surgery.

Table 3. Details of Patients not Requiring Surgery

\begin{tabular}{lcccccc}
\hline \multicolumn{1}{c}{ Surgery } & ERM & Schisis & PTMH & FTMH & PRD & Overall \\
\hline No & 96 & 41 & 19 & 15 & 10 & 181 \\
Baseline logMAR & 0.40 & 0.32 & 0.40 & 0.39 & 1.10 & 0.35 \\
End of F/U logMAR & 0.41 & 0.38 & 0.34 & 0.40 & 1.37 & 0.36 \\
Baseline MP1 (dB) & 15.5 & 12.6 & 14.2 & 12.4 & 11.6 & 14.2 \\
End of F/U MP1 (dB) & 16.2 & 14.4 & 14.5 & 12.8 & 10.2 & 14.9 \\
Baseline fixation & $89 \mathrm{~S}, 7 \mathrm{R}$ & $33 \mathrm{~S}, 8 \mathrm{R}$ & $13 \mathrm{~S}, 6 \mathrm{R}$ & $7 \mathrm{~S}, 6 \mathrm{R}, 2 \mathrm{U}$ & $8 \mathrm{~S}, 2 \mathrm{R}$ & $150 \mathrm{~S}, 29 \mathrm{R}, 2 \mathrm{U}$ \\
End of F/U fixation & $89 \mathrm{~S}, 7 \mathrm{R}$ & $33 \mathrm{~S}, 8 \mathrm{R}$ & 13S, 6R & 8S, 7R & $8 \mathrm{~S}, 2 \mathrm{R}$ & $151 \mathrm{~S}, 30 \mathrm{R}$ \\
\hline
\end{tabular}

LogMAR, logarithm of the minimum angle of resolution; $F / U$, follow-up; $S$, stable; $R$, relatively stable; $U$, unstable. 
Table 4. Spearman Correlation Between Fixation Stability Classes (Stable, Relatively Stable, Unstable), Foveal Sensitivity Difference Between Baseline and Preoperative Visits (or End of Follow-up Visit), and Need for Surgery

\begin{tabular}{|c|c|c|c|c|c|c|}
\hline & Group & $\begin{array}{c}\text { Fix } \\
t_{0}\end{array}$ & $\begin{array}{l}d B \\
t_{0}\end{array}$ & $\begin{array}{c}\text { Delta } \\
\mathrm{dB}\end{array}$ & $\begin{array}{l}\text { Delta } \\
\text { Fix }\end{array}$ & Surgery \\
\hline Group & - & $0.000^{*}$ & $0.000^{\star}$ & 0.343 & $0.000^{*}$ & $0.001^{*}$ \\
\hline Fix $_{0}$ & $0.000^{\star}$ & - & $0.000^{\star}$ & 0.654 & 0.609 & 0.229 \\
\hline$d B t_{0}$ & $0.000^{\star}$ & $0.000^{*}$ & - & 0.165 & 0.106 & 0.955 \\
\hline Delta dB & $0.343^{\star}$ & 0.654 & 0.165 & - & $0.000^{*}$ & $0.000^{\star}$ \\
\hline Delta fix & $0.000^{\star}$ & 0.609 & 0.106 & $0.000^{*}$ & - & $0.000^{\star}$ \\
\hline Surgery & 0.001 & 0.229 & 0.955 & $0.000^{\star}$ & $0.000^{\star}$ & - \\
\hline
\end{tabular}

Significance has been set at the 0.01 level. Patients in the PRD group who underwent surgery right after baseline visit have been excluded.

${ }^{*}$ Correlation is significant at the 0.01 level (2 tailed).

$d B t_{0}$, foveal sensitivity in $d B$ at $t_{0}$; delta $d B$, difference in $d B$ between baseline examination and preoperative visit (for operated eyes) or end of follow-up visit (for stable eyes that did not undergo surgery); delta fix, difference in class of fixation stability (stable, relatively stable, unstable) between baseline examination and preoperative visit (for operated eyes) or end of follow-up visit (for stable eyes that did not undergo surgery); fix $t_{0}$, fixation at $t_{0}$; surgery, need for surgery (yes, no).

\section{PRD Patient No. 3}

A totally asymptomatic 36-year-old woman (visual acuity 20/32; sph -22; foveal sensitivity $11 \mathrm{~dB}$, stable fixation at baseline) presented for routine examination. Optical coherence tomography showed an ERM connected with the detached retina, and retinal detachment included superotemporal vascular arcades. Three months later, visual acuity dropped to 20/200 with relatively stable fixation and underwent PPV, cortical vitreous peel, ILM peel, and silicone oil tamponade $(\mathrm{SiO})$ with good success and regained a stable fixation and 20/50 visual acuity (Table 2).

\section{PRD Patient No. 13}

A 48-year-old man presented with $\mathrm{MH}$ associated to PRD, unstable fixation, and 20/400 vision (-22 dpt; 5 $\mathrm{dB}$ foveal sensitivity; Figure 2A). Despite immediate PPV with ILM peel and SiO tamponade, 1 month postoperatively, the retina did not flatten completely on the PS (Figure 2B), visual acuity was unchanged, and fixation was unstable. Episcleral macular buckle was performed with success (Figure 2C), visual acuity increased to $20 / 80$ and foveal sensitivity to $9 \mathrm{~dB}$ with stable fixation.

\section{Discussion}

Pathologic myopia with PS is consistently associated to posterior vitreoretinal alterations with protean presentation: more that one half of our patients showed frank vitreoretinal abnormalities in the staphyloma and bilateral involvement represented almost the rule: 4 of 5 cases. Shimada et $\mathrm{al}^{11}$ also reported a very high prevalence of paravascular retinal cysts $(49.5 \%)$, vascular microfolds $(44.6 \%)$, and paravascular retinal
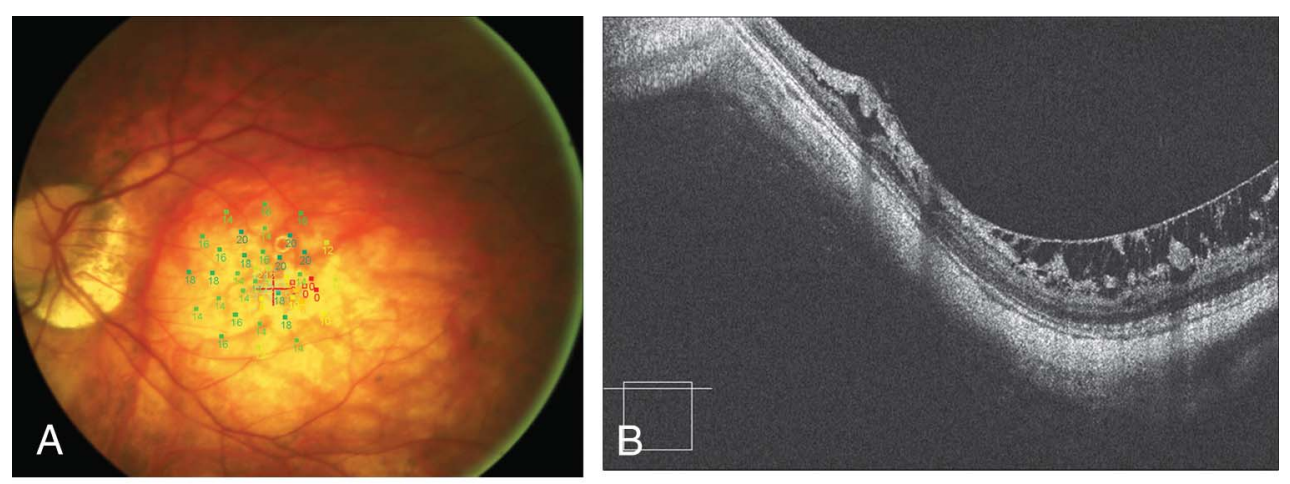

Fig. 1. A. Schisis patient no.43: Microperimetry shows foveal sensitivity of $12 \mathrm{~dB}$ with stable fixation and an area of dense scotoma. B. Schisis patient no. 43: Spectral domain optical coherence tomographic scan shows loss of the foveal contour and increase of the retinal thickness with schisis-like changes of the parafoveal temporal inner retinal layers, represented at optical coherence tomographic scan by large intraretinal hyporeflective spaces with vertical column-like reflective strands of neuroretinal tissue. C. Schisis patient no. 43: Six months later, MP measures $10 \mathrm{~dB}$ in fovea, with relatively stable fixation and an enlargement of the area of dense scotoma. D. Schisis patient no. 43: Six months later, spectral domain optical coherence tomographic scan shows a further increase of the central retinal thickness with a large subfoveal cystoid hyporeflective space determining a foveal detachment, a wider inner retinal schisis temporally to the fovea and the formation of outer retinal schisis nasally to the fovea. 

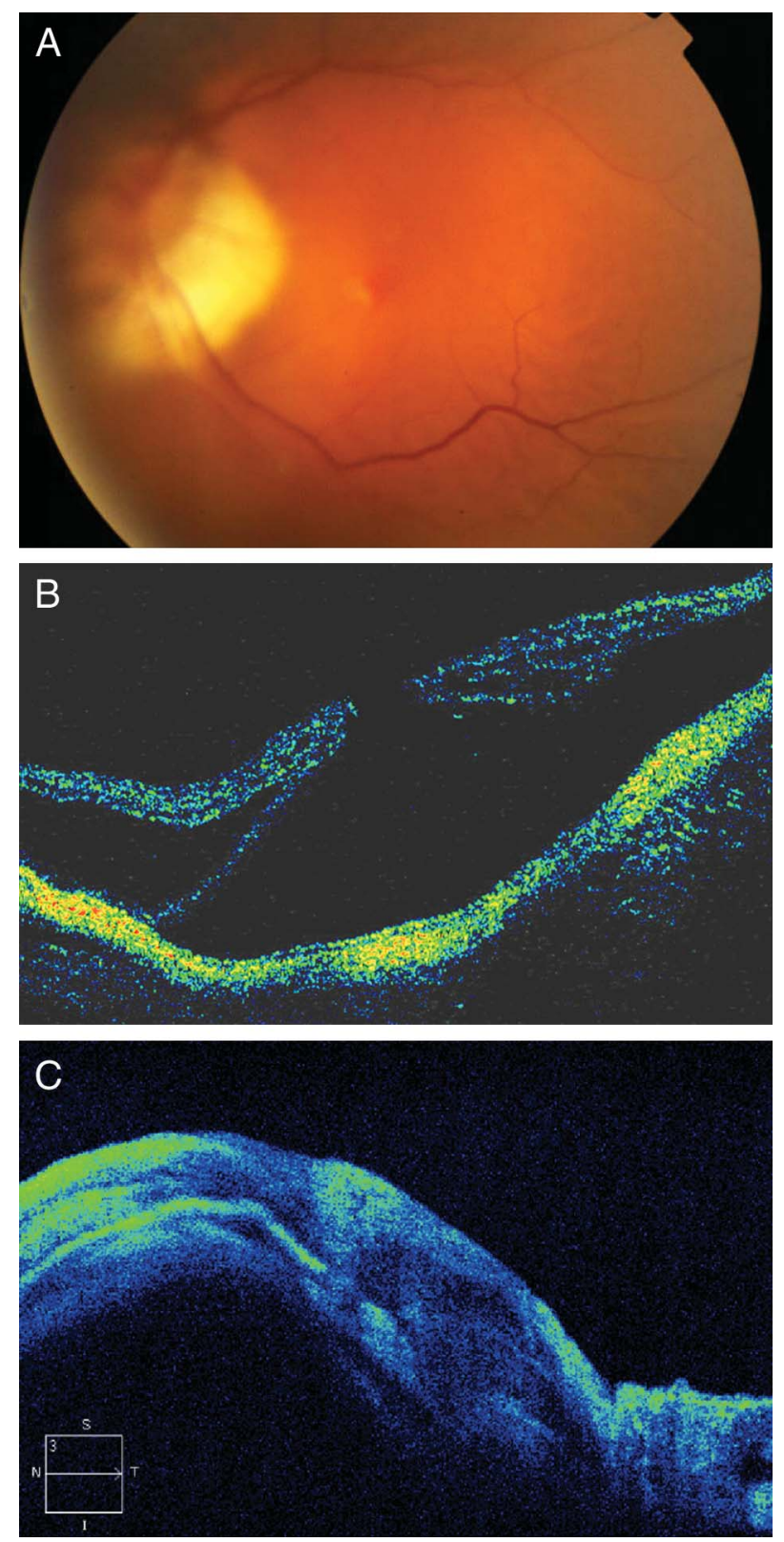

Fig. 2. A. PRD patient no. 13: Fundus retinography of posterior retinal detachment with MH. B. PRD patient no. 13: One month after PPV, time domain optical coherence tomographic scan shows a residual, shallow retinal detachment with an opened $\mathrm{MH}$ and the presence of a large, parafoveal hyporeflective intraretinal space consisting with the presence of an outer retinal schisis. C. PRD patient no. 13: After macular buckling surgical procedure, spectral domain optical coherence tomography shows a homogeneous reflectivity of the neuroretinal layers with disappearance of the area of retinal schisis, a convex shape of the macular profile because of the scleral indentation of the macular buckle, a flattened retina, and the closure of the macula hole.

holes $(26.8 \%)$, most of which are asymptomatic, while $\mathrm{Wu}$ et $\mathrm{al}^{6}$ recorded a lower prevalence of foveoschisis $(8 \%)$ although recognizing a marked increase for eyes longer than $30 \mathrm{~mm}$. Posterior retinal detachment, often asymptomatic, is also relatively common, having been described in up to $9 \%$ of eyes with PS. ${ }^{12}$

We detected a slightly higher prevalence of vitreoretinal abnormalities, probably because of more restrictive inclusion criteria that selected longer bulbs. A selection bias could also be invoked in the setting of a tertiary referral center specializing in the care of myopia, and the extensive use of OCT in asymptomatic patients might have contributed to a higher detection rate in asymptomatic patients.

The progressive elongation of PS has been well documented $^{2,13,14}$ also as a risk factor for disease progression and unfavorable prognosis; Green and Grossnicklaus ${ }^{15}$ described a posterior schisis variant, which they named "stretch schisis," where scleral elongation would be the main reason for retinal layer separation, regardless of vitreous traction. They described it as "... a condition that results from retinal vessels that do not lengthen as much as the underlying neurosensory retina, resulting in a schisis-like configuration...." We indeed observed a similar picture in 7 eyes with no obvious ERM, and although we cannot infer this as a separate entity, it did not seem to follow the schisis model theorized by Sun et al. ${ }^{16}$ An alternative explanation could be invoked as also in the absence of ERM, differential compliance of inner and outer retinal aspects, noncompliant ILM, "stiff retinal vessels" and/or cortical vitreous remnants could result in neurosensory detachment limited to the staphyloma. ${ }^{17}$

Although excellent studies exist on the natural history of myopic maculopathy, ${ }^{2,14}$ less is known about the risk of progression of vitreoretinal interface pathology associated to PM with PS and the need for surgery. Gaucher et $\mathrm{al}^{18}$ reported macular schisis progression in 20 of 29 eyes (68\%) after 31 months of mean follow-up, half of which associated with ERM and operated 11 of them with significant improvement.

Only a small percentage of our patients eventually required treatment (15\%, Table 2$)$, despite a lengthy follow-up; indeed, the true progression rate was even lower if we consider that 9 PRD eyes underwent surgery upon presentation, thus lowering the figure to $11.7 \%$ of the entire sample $(33-9=24 ; 24 / 205=$ $0.117)$. Progression patterns varied significantly across groups (Table 2): Risk of progression almost doubled for Schisis and PTMH and reached 50\% for symptomatic Schisis while ERM chances of requiring surgery were approximately one tenth of the average (2\%). It should be emphasized that both "progression" and "need for surgery" largely depend on individual judgment and as such are largely debatable. In the FTMH group for instance, we elected to withhold surgery in symptomatic patients with extensive choroidal 
atrophy, in the absence of PRD. It is our experience that such patients rarely experience $\mathrm{MH}$ closure after surgery and only occasionally improve vision.

Surgical techniques, both vitreous ${ }^{19}$ and scleral buckling procedures, have been thoroughly investigated. ${ }^{20}$ Ando et $\mathrm{al}^{20}$ reported favorable outcome in $93 \%$ of cases after EMB limited to MH cases, while Baba et $\mathrm{al}^{21}$ reported a broader use of EMB for posterior schisis without $\mathrm{MH}$, obtaining a $66 \%$ success rate. We performed PPV as a primary procedure in all patients, with a primary anatomical success rate of $60.6 \%$ (20 of 33). When secondary surgery was deemed necessary, EMB was performed, with increase in foveal sensitivity and fixation stability in most cases (Table 2). The rationale for primary PPV, ${ }^{22}$ leaving EMB as a secondary option, is based on the presence of an obvious vitreous traction in most patients that can be effectively relieved by means of vitrectomy.

Failure to achieve complete foveal reattachment after primary PPV may point to a relative insufficiency of the retina (or inner retinal layers) compared with the scleral bed. Alternatively, ILM and/or vitreous cortex remnants can still cause retinal contraction, and their removal would allow retinal distension. A secondary buckling procedure, limited to those cases, would take care of a relative "retinal shortness" and counteract residual contraction because of hidden ILM and/or cortex islets. Episcleral macular buckle would also work where a "stretch schisis" mechanism postulated by Green coexists.

Foveal sensitivity decrease and worsening of fixation stability were significantly associated to the need for surgery (Table 4). Although the retrospective nature of our study obviously introduced a bias, because a drop in visual acuity is often associated to both fixation instability and loss of foveal sensitivity, we also observed patients who complained of "blurred vision" before Snellen acuity dropped (Schisis patient no. 43 and PTMH patient no. 11). In such patients, MP showed altered fixation and decreased foveal sensitivity, weeks before acuity worsened. It is conceivable that fixation is subtly altered by the initial photoreceptor damage although the patient's ability to read the Snellen chart remains stable until further damage consistently impairs fixation, impacting visual acuity.

Present study suffers from the many limitations of retrospective series: lack of data systematization and, at time, consistency. Vitreoretinal interface alterations in highly myopic eyes have not been univocally classified yet and both disease progression and surgical indications are largely debatable and deserve standardization. We believe that the strength of present study resides in the largeness of series, the lengthy followup, and single-surgeon management.
In summary, PS in PM is associated to a variety of vitreoretinal interface modifications whose natural history greatly varies. Foveal sensitivity and fixation patterns are associated to the risk of progression, and visual prognosis differs among groups. We believe the many presentations we describe do not just represent penetration variability of one disease but point at separate conditions that warrant diversified treatment.

Key words: high myopia, posterior staphyloma, macular pathologies, surgery.

\section{References}

1. Curtin BJ. The Myopias: Basic Sciences and Clinical Management. Philadelphia, PA: Harper \& Row; 1985:7-9.

2. Saka N, Ohno-Matsui K, Shimada N, et al. Long-term changes in axial length in adult eyes with pathologic myopia. Am J Ophthalmol 2010;150:562-568.e1.

3. Curtin BJ. The posterior staphyloma of pathologic myopia. Trans Am Ophthalmol Soc 1977;75:67-86.

4. Takano M, Kishi S. Foveal retinoschisis and retinal detachment in severely myopic eyes with posterior staphyloma. Am J Ophthalmol 1999;128:472-476.

5. Witkin AJ, Ko TH, Fujimoto JG, et al. Redefining lamellar holes and the vitreomacular interface: an ultrahigh-resolution optical coherence tomography study. Ophthalmology 2006;113:388-397.

6. Wu PC, Chen YJ, Chen YH, et al. Factors associated with foveoschisis and foveal detachment without macular hole in high myopia. Eye (Lond) 2009;23:356-361.

7. Hayashi K, Ohno-Matsui K, Shimada N, et al. Long-term pattern of progression of myopic maculopathy: a natural history study. Ophthalmology 2010;117:1595-1611, 1611.e1-e4.

8. Richter-Mueksch S, Vecsei-Marlovits P, Sacu SG, et al. Functional macular mapping in patients with vitreomacular pathologic features before and after surgery. Am J Ophthalmol 2007; 144:23-31.

9. Puliafito CA, Hee MR, Lin CP, et al. Imaging of macular diseases with optical coherence tomography. Ophthalmology 1995;102:217-229.

10. Ripandelli G, Coppè AM, Parisi V, Stirpe M. Fellow eye findings in highly myopic subjects operated for retinal detachment associated with a macular hole. Ophthalmology 2008;115: 1489-1493.

11. Shimada N, Ohno-Matsui K, Nishimuta A, et al. Detection of paravascular lamellar holes and other paravascular abnormalities by optical coherence tomography in eyes with high myopia. Ophthalmology 2008;115:708-717.

12. Baba T, Ohno-Matsui K, Futagami S, et al. Prevalence and characteristics of foveal retinal detachment without macular hole in high myopia. Am J Ophthalmol 2003;135:338-342.

13. Stirpe M, Michels RG. Retinal detachment in highly myopic eyes due to macular holes and epiretinal traction. Retina 1990; 10:113-114.

14. Vongphanit J, Mitchell P, Wang JJ. Prevalence and progression of myopic retinopathy in an older population. Ophthalmology 2002;109:704-711.

15. Green WR, Grossnicklaus HE. Histopathologic features of high myopia. In: Midena E, ed. Myopia and Related Diseases, VI International Congress Fondazione G. B. Bietti per l'Oftalmologia. New York, New York: Ophthalmic Communications Society, Inc; 2005:48. 
16. Sun CB, Liu Z, Xue AQ, Yao K. Natural evolution from macular retinoschisis to full-thickness macular hole in highly myopic eyes. Eye (Lond) 2010;24:1787-1791.

17. Sayanagi K, Ikuno Y, Tano Y. Tractional internal limiting membrane detachment in highly myopic eyes. Am J Ophthalmol 2006;142:850-852.

18. Gaucher D, Haouchine B, Tadayoni R, et al. Long-term follow-up of high myopic foveoschisis: natural course and surgical outcome. Am J Ophthalmol 2007;143: $455-462$.

19. Chang S. Vitrectomy for traction macular detachment and retinoschisis in highly myopic eyes. In: Midena E, ed. Myopia and Related Diseases, VI International Congress Fondazione
G. B. Bietti per l'Oftalmologia. New York, New York: Ophthalmic Communications Society, Inc; 2005:195-199.

20. Ando F, Ohba N, Touura K, Hirose H. Anatomical and visual outcomes after episcleral macular buckling compared with those after pars plana vitrectomy for retinal detachment caused by macular hole in highly myopic eyes. Retina 2007;27:37-44.

21. Baba T, Tanaka S, Maesawa A, et al. Scleral buckling with macular plombe for eyes with myopic macular retinoschisis and retinal detachment without macular hole. Am J Ophthalmol 2006;142:483-487.

22. Seike C, Kusaka S, Sakagami K, Ohashi Y. Reopening of macular holes in highly myopic eyes with retinal detachments. Retina 1997;17:2-6. 